\title{
DOMAIN DECOMPOSITION ALGORITHMS FOR MIXED METHODS FOR SECOND-ORDER ELLIPTIC PROBLEMS
}

\author{
ZHANGXIN CHEN, RICHARD E. EWING, AND RAYTCHO LAZAROV
}

\begin{abstract}
In this paper domain decomposition algorithms for mixed finite element methods for linear second-order elliptic problems in $\mathbb{R}^{2}$ and $\mathbb{R}^{3}$ are developed. A convergence theory for two-level and multilevel Schwarz methods applied to the algorithms under consideration is given. It is shown that the condition number of these iterative methods is bounded uniformly from above in the same manner as in the theory of domain decomposition methods for conforming and nonconforming finite element methods for the same differential problems. Numerical experiments are presented to illustrate the present techniques.
\end{abstract}

\section{INTRODUCTION}

This is the second paper of a sequence where we develop and analyze efficient iterative algorithms for solving the linear system arising from mixed finite element methods for linear and quasilinear second-order elliptic problems in $\mathbb{R}^{2}$ and $\mathbb{R}^{3}$. In the first paper [12], a new approach for developing multigrid algorithms for the mixed finite element methods was introduced. It was first shown that the mixed finite element formulation can be algebraically condensed to a symmetric and positive definite system for Lagrange multipliers using the features of the existing mixed finite element spaces. It was then proven that optimal multigrid algorithms can be designed for the resulting symmetric and positive definite system, which exactly corresponds to the system arising from certain nonconforming finite element methods. The advantages of this approach are that the convergence analysis for the multigrid algorithms with the $\mathcal{V}$-and $\mathcal{W}$-cycles for general second-order elliptic problems with a tensor coefficient can be given, and that these multigrid algorithms can be easily implemented.

It has been known that, owing to its saddle point property, it is difficult to develop efficient domain decomposition methods for solving the linear system generated by the mixed finite element approximation of second-order elliptic problems. There have been two types of substructuring domain decomposition methods for the mixed methods so far. The first method is based on a substructuring method for the flux variable (the gradient of the scalar unknown times the coefficient of the differential problems) on the space of divergence-free vectors. This approach is limited to two space dimensions $[24,25,26,27,28,31,32,40]$. The other method

Received by the editor August 2, 1994 and, in revised form, March 21, 1995.

1991 Mathematics Subject Classification. Primary 65N30, 65N22, 65F10.

Key words and phrases. Finite element, implementation, mixed method, conforming and nonconforming methods, domain decomposition, convergence, projection of coefficient.

Partly supported by the Department of Energy under contract DE-ACOS-840R21400. 
is the so-called dual variable method $[19,16,18,27,28]$. This approach makes use of a discretization of the flux operator (the coefficient times the gradient), which transfers the original saddle point problem to an elliptic problem for the scalar unknown and its approximations over edges or faces, i.e., the Lagrange multipliers, by eliminating the flux variable. Namely, the first approach is proposed in terms of domain decomposition methods for a positive definite problem for the flux variable on the space of divergence-free vectors, while the second approach is established on the domain decomposition methods for a positive definite problem for the scalar and Lagrange multiplier. Recently, an iterative procedure based on domain decomposition techniques [21] was proposed for solving the linear system for the scalar, the flux, and the Lagrange multiplier, but the convergence analysis is restricted to use of subdomains as small as individual finite elements.

Our objective in this paper is to develop domain decomposition algorithms for mixed finite element methods based on the approach described in [12]. The algorithms are based on domain decomposition methods for the Lagrange multiplier variable only, and thus differ from the approaches summarized above. The main advantages of our approach are that it works for two and three space dimension problems, and the dimension of the linear system for which the domain decomposition algorithms are designed to solve is the smallest among all the existing approaches. Also, unlike to the elimination process in $[19,16,18,25,26,31,32]$, where the elimination is globally done from the original linear system of the mixed finite element discretization, the elimination procedure is here carried out in terms of an algebraical, element-by-element condensation, which uses the features of the known mixed finite element spaces and does not need to introduce any extra operators. This process generates a linear system which can be naturally obtained from the nonconforming finite element approximation of the same differential problems. As a consequence, the standard theory for the domain decomposition methods applied to nonconforming (even conforming) finite element methods applies to the mixed methods. Finally, bubble functions have been used in $[1,2,10]$ to establish the equivalence between mixed finite element methods and certain nonconforming methods. The approach under consideration does not make use of bubble functions. The present approach is exploited for the first time to design domain decomposition algorithms for mixed methods.

In the next section we introduce the continuous problem and its mixed finite element discretization. Then, in $\S 3$ two-level and multilevel Schwarz algorithms for the mixed finite elements on triangles are considered. An abstract convergence theory is established in a rather general setting. It is proven that the condition number of the Schwarz methods is bounded uniformly from above in the same manner as in the theory of domain decomposition methods for conforming and nonconforming methods for the same differential problems. Specific examples are given to verify the abstract theory. In $\S 4$, we show that the same algorithm and analysis can be carried out for the mixed methods on rectangles. Their extensions to simplexes, rectangular parallelepipeds, and prisms are given in $\S 5, \S 6$, and $\S 7$, respectively. The overall convergence analysis is carried out as follows. We first analyze the domain decomposition method for the nonconforming finite element method, and then apply the resulting analysis for the mixed method. Also, a detailed analysis is given for triangles and simplexes, and the analysis for rectangular parallelepipeds and prisms follows from the triangular case by establishing certain isomorphisms 
between the triangular and rectangular elements. Finally, numerical experiments are given in $\S 8$ to illustrate the present theory.

\section{Mixed Finite Element methods}

Let $\Omega$ be a bounded domain in $\mathbb{R}^{d}, d=2$ or 3 , with the polygonal boundary $\partial \Omega$. We consider the elliptic problem

$$
\begin{array}{ll}
-\nabla \cdot(\mathcal{A} \nabla u)=f & \text { in } \Omega, \\
u=0 & \text { on } \partial \Omega,
\end{array}
$$

where $\mathcal{A}(x)$ is a uniformly positive definite, bounded, symmetric tensor and $f(x) \in$ $L^{2}(\Omega)\left(H^{k}(\Omega)=W^{k, 2}(\Omega)\right.$ is the Sobolev space of $k$ times differentiable functions in $L^{2}(\Omega)$ ). Let $(\cdot, \cdot)_{S}$ denote the $L^{2}(S)$ inner product (we omit $S$ if $S=\Omega$ ), and let

$$
\begin{aligned}
& V=H(\operatorname{div} ; \Omega)=\left\{v \in\left(L^{2}(\Omega)\right)^{d}: \nabla \cdot v \in L^{2}(\Omega)\right\}, \\
& W=L^{2}(\Omega) .
\end{aligned}
$$

Then (2.1) is formulated in the following mixed form for the pair $(\sigma, u) \in V \times W$ :

$$
\begin{array}{ll}
(\nabla \cdot \sigma, w)=(f, w), & \forall w \in W, \\
\left(\mathcal{A}^{-1} \sigma, v\right)-(u, \nabla \cdot v)=0, & \forall v \in V .
\end{array}
$$

It can be easily seen that (2.1) is equivalent to (2.2) through the relation

$$
\sigma=-\mathcal{A} \nabla u
$$

To define a finite element method, we need a partition $\mathcal{E}_{h}$ of $\Omega$ into elements $E$, say, simplexes, rectangular parallelepipeds, and/or prisms. In $\mathcal{E}_{h}$, we also need that adjacent elements completely share their common edge or face; let $\partial \mathcal{E}_{h}$ denote the set of all interior edges $(d=2)$ or faces $(d=3) e$ of $\mathcal{E}_{h}$.

Let $V_{h} \times W_{h} \subset V \times W$ denote some standard mixed finite element space for second-order elliptic problems defined over $\mathcal{E}_{h}$ (see, e.g., [6, 7, 8, 14, 22, 34, 35, 36]). This space is finite-dimensional and defined locally on each element $E \in \mathcal{E}_{h}$; so let $V_{h}(E)=\left.V_{h}\right|_{E}$ and $W_{h}(E)=\left.W_{h}\right|_{E}$. The constraint $V_{h} \subset V$ says that the normal component of the members of $V_{h}$ is continuous across the interior boundaries in $\partial \mathcal{E}_{h}$. Following [2], we relax this constraint on $V_{h}$ by defining

$$
\tilde{V}_{h}=\left\{v \in L^{2}(\Omega):\left.v\right|_{E} \in V_{h}(E) \text { for each } E \in \mathcal{E}_{h}\right\} .
$$

We then need to introduce Lagrange multipliers to enforce the required continuity on $\tilde{V}_{h}$, so define

$$
L_{h}=\left\{\mu \in L^{2}\left(\bigcup_{e \in \partial \mathcal{E}_{h}} e\right):\left.\left.\mu\right|_{e} \in V_{h} \cdot \nu\right|_{e} \text { for each } e \in \partial \mathcal{E}_{h}\right\},
$$


where $\nu$ is the unit normal to $e$. The hybrid form of the mixed method for (2.1) is to find $\left(\sigma_{h}, u_{h}, \lambda_{h}\right) \in \tilde{V}_{h} \times W_{h} \times L_{h}$ such that

$$
\begin{aligned}
& \sum_{E \in \mathcal{E}_{h}}\left(\nabla \cdot \sigma_{h}, w\right)_{E}=(f, w), \quad \forall w \in W_{h}, \\
& \left(\mathcal{B}_{h} \sigma_{h}, v\right)-\sum_{E \in \mathcal{E}_{h}}\left[\left(u_{h}, \nabla \cdot v\right)_{E}-\left(\lambda_{h}, v \cdot \nu_{E}\right)_{\partial E \backslash \partial \Omega}\right]=0, \quad \forall v \in \tilde{V}_{h}, \\
& \sum_{E \in \mathcal{E}_{h}}\left(\sigma_{h} \cdot \nu_{E}, \mu\right)_{\partial E \backslash \partial \Omega}=0, \quad \forall \mu \in L_{h},
\end{aligned}
$$

where $\mathcal{B}_{h}=P_{h} \mathcal{A}^{-1}$ (component-by-component) and $P_{h}$ is the $L^{2}$-projection onto $W_{h}$. Note that $(2.4 \mathrm{c})$ enforces the continuity requirement mentioned above, so in fact $\sigma_{h} \in V_{h}$. Also, $(2.4)$ has a unique solution $[2,10]$. Finally, the projected mixed finite element method is used here. The reason for this is that this projected version produces a much simpler linear system than the usual mixed method, as shown in [12]. We emphasize that the present theory applies to the usual mixed method since the convergence analysis for both cases are the same; for more information on the relationship between the usual and projected mixed methods, refer to [12]. The next six sections are devoted to designing domain decomposition algorithms for solving the linear system arising from (2.4).

\section{Triangular CASE}

In this and the next sections we consider the two-dimensional case. We first analyze the lowest-order Raviart-Thomas space [36] (equivalently, the lowest-order Brezzi-Douglas-Marini space [8]) on triangles.

3.1. Linear system of algebraic equations. The lowest-order Raviart-Thomas space [36] over triangles is defined by

$$
\begin{aligned}
& V_{h}(E)=\left(P_{0}(E)\right)^{2} \oplus\left((x, y) P_{0}(E)\right), \\
& W_{h}(E)=P_{0}(E) \\
& L_{h}(e)=P_{0}(e)
\end{aligned}
$$

where $P_{i}(E)$ is the restriction of the set of all polynomials of total degree not bigger than $i \geq 0$ to the set $E$. Let $f_{h}=P_{h} f, J_{h}^{f}=f_{h}(x, y) / 2$, and $\mathcal{B}_{h}=\left(\alpha_{i j}\right)$. Then it is shown [12] that the $\lambda_{h}$ from (2.4) satisfies the equation (3.1) below.

Lemma 1. Let

$$
\begin{aligned}
& M_{h}(\chi, \mu)=\sum_{E \in \mathcal{E}_{h}}\left(\chi, \nu_{E}\right)_{\partial E} \beta^{E}\left(\mu, \nu_{E}\right)_{\partial E}, \quad \chi, \mu \in L_{h}, \\
& F_{h}(\mu)=-\sum_{E \in \mathcal{E}_{h}} \frac{\left(J_{h}^{f}, 1\right)_{E}}{|E|} \cdot\left(\mu, \nu_{E}\right)_{\partial E}+\sum_{E \in \mathcal{E}_{h}}\left(\mu J_{h}^{f}, \nu_{E}\right)_{\partial E}, \quad \mu \in L_{h},
\end{aligned}
$$

where $\beta^{E}=\left(\beta_{i j}^{E}\right)=\left(\left(\alpha_{i j}, 1\right)_{E}\right)^{-1}, \nu_{E}$ is the outer unit normal to $E$, and $|E|$ denotes the area of $E$. Then $\lambda_{h} \in \mathcal{L}_{h}$ satisfies

$$
M_{h}\left(\lambda_{h}, \mu\right)=F_{h}(\mu), \quad \forall \mu \in \mathcal{L}_{h},
$$


where

$$
\mathcal{L}_{h}=\left\{\mu \in L_{h}:\left.\mu\right|_{e}=0 \text { for each } e \subset \partial \Omega\right\} .
$$

Let the basis in $L_{h}$ be chosen as usual. Namely, take $\mu=1$ on one edge and $\mu=0$ elsewhere in (3.1). Then it follows from (3.1) that the contributions of each triangle $E$ to the stiffness matrix and the right-hand side are

$$
m_{i j}^{E}=\bar{\nu}_{E}^{i} \beta^{E} \bar{\nu}_{E}^{j}, \quad F_{i}^{E}=-\frac{\left(J_{h}^{f}, \bar{\nu}_{E}^{i}\right)_{E}}{|E|}+\left(J_{h}^{f}, \nu_{E}^{i}\right)_{e_{E}^{i}}
$$

where $\bar{\nu}_{E}^{i}=\left|e_{E}^{i}\right| \nu_{E}^{i}$ and $\left|e_{E}^{i}\right|$ is the length of the edge $e_{E}^{i}$. Hence, we obtain the linear system for $\lambda_{h}$ :

$$
M \lambda=F,
$$

where $M=\left(m_{i j}\right), \lambda$ is the vector of degrees of freedom of $\lambda_{h}$, and $F=\left(F_{i}\right)$.

The following lemma [12] says that (3.3) can also be obtained from the $P_{1}$ nonconforming finite element method.

Lemma 2. Let

(3.4) $N_{h}=\left\{v \in L^{2}(\Omega):\left.v\right|_{E} \in P_{1}(E), \forall E \in \mathcal{E}_{h} ; v\right.$ is continuous at the midpoints of interior sides and vanishes at the midpoints of sides on $\partial \Omega\}$.

Then (3.3) corresponds to the linear system arising from the problem: Find $\psi_{h} \in N_{h}$ such that

$$
a_{h}\left(\psi_{h}, \varphi\right)=\left(f_{h}, \varphi\right), \quad \forall \varphi \in N_{h}
$$

where $a_{h}\left(\psi_{h}, \varphi\right)=\sum_{E \in \mathcal{E}_{h}}\left(\mathcal{B}_{h}^{-1} \nabla \psi_{h}, \nabla \varphi\right)_{E}$.

The equivalence stated in Lemma 2 is used to develop the domain decomposition algorithm for (3.3).

After the computation of $\lambda_{h}$, we can easily calculate $\sigma_{h}$ and $u_{h}$ from (2.4) if they are needed. For each $E$ in $\mathcal{E}_{h}$, set $\left.\sigma_{h}\right|_{E}=\left(a_{E}^{1}+b_{E} x, a_{E}^{2}+b_{E} y\right)$. It follows [12] that

$$
\begin{aligned}
a_{E}^{j}= & -\left.\sum_{i=1}^{3}\left|e_{E}^{i}\right|\left(\beta_{j 1}^{E} \nu_{E}^{i(1)}+\beta_{j 2}^{E} \nu_{E}^{i(2)}\right) \lambda_{h}\right|_{e_{E}^{i}} \\
& -\frac{f_{E}}{2} \sum_{i=1}^{2}\left(\beta_{j i}^{E}\left(\alpha_{i 1} x+\alpha_{i 2} y\right), 1\right)_{E}, \quad j=1,2, \\
b_{E}= & \frac{f_{E}}{2}
\end{aligned}
$$

where $f_{E}=\left.f_{h}\right|_{E}$ and $\nu_{E}^{i}=\left(\nu_{E}^{i(1)}, \nu_{E}^{i(2)}\right)$, and that

$$
\left.u_{h}\right|_{E}=\frac{1}{2|E|}\left(\left(\mathcal{B}_{h} \sigma_{h},(x, y)\right)_{E}+\left.\sum_{i=1}^{3} \lambda_{h}\right|_{e_{E}^{i}}\left((x, y), \nu_{E}^{i}\right)_{e_{E}^{i}}\right) \text {. }
$$

We end this subsection with three remarks about (3.3). First, there are at most five nonzero entries per row in the stiffness matrix $M$. Second, it is easy to see that the matrix $M$ is a symmetric and positive definite matrix; moreover, if the angles of every $E$ in $\mathcal{E}_{h}$ are not bigger than $\pi / 2$, then it is an $M$-matrix. Finally, while (3.3) can be obtained by means of the usual approach [12], the present approach is much simpler. 
3.2. Two-level additive Schwarz method. We now develop a two-level additive Schwarz algorithm for (3.3). We need to assume a structure to our family of partitions. In the first step, let $\mathcal{E}_{H}$ be a quasi-regular coarse triangulation [15] of $\Omega$ into nonoverlapping triangular substructures $\Omega_{i}, i=1, \ldots, n$. Then, in the second step we refine $\mathcal{E}_{H}$ into triangles to have a quasi-regular triangulation $\mathcal{E}_{h}$. Finally, let $\left\{\Omega_{i}^{\prime}\right\}_{i=1}^{n}$ be an overlapping domain decomposition of $\Omega$ by extending $\Omega_{i}$ with the overlap parameter $\delta$. The decomposition is assumed to align with the boundary $\partial \Omega$, and the parameter $\delta$ is defined by $\delta=\min \left\{\operatorname{dist}\left(\partial \Omega_{i} \backslash \partial \Omega, \partial \Omega_{i}^{\prime} \backslash \partial \Omega\right), i=1, \ldots, n\right\}$. Associated with each $\Omega_{i}^{\prime}$, let $N_{h}^{i}$ be the $P_{1}$ nonconforming finite element space whose elements have support in $\Omega_{i}^{\prime}$, as defined in (3.4). The finite element space $N_{h}$ is represented as a sum of $n+1$ subspaces:

$$
N_{h}=N_{h}^{0}+N_{h}^{1}+\ldots+N_{h}^{n},
$$

where the coarse space $N_{h}^{0}$ will be defined later. We now define the operators $\Pi_{i}: N_{h} \rightarrow N_{h}^{i}, i=0,1, \ldots, n$, by

$$
a_{h}\left(\Pi_{i} v, w\right)=a_{h}(v, w), \quad \forall w \in N_{h}^{i},
$$

and the operator $\Pi: N_{h} \rightarrow N_{h}$ by

$$
\Pi=\sum_{i=0}^{n} \Pi_{i} .
$$

Two-level additive algorithm. The additive Schwarz algorithm for (3.3) is given by

$$
\Pi \psi_{h}=\widehat{f}_{h}, \quad \widehat{f}_{h}=\sum_{i=0}^{n} f_{i}
$$

where $f_{i}$ satisfies

$$
a_{h}\left(f_{i}, v\right)=\left(f_{h}, v\right), \quad \forall v \in N_{h}^{i}, i=0,1, \ldots, n .
$$

Note that (3.5) and (3.11) have the same solution and thus produce the same system (3.3).

3.2.1. Convergence theory. We now develop an abstract convergence theory for bounds on the condition number of $\Pi$. Specific examples to which the abstract theory applies will be given in the next subsection. Following Dryja and Widlund's framework [23], the abstract theory is written in terms of the following two assumptions:

(A1) There is a constant $\mathcal{C}$ such that every $v \in N_{h}$ can be represented by $v=$ $\sum_{i=0}^{n} v_{i}$ with $v_{i} \in N_{h}^{i}$ satisfying

$$
\sum_{i=0}^{n} a_{h}\left(v_{i}, v_{i}\right) \leq \mathcal{C} a_{h}(v, v) .
$$

(A2) Let $\kappa=\left(\kappa_{i j}\right)$ be a symmetric matrix with $\kappa_{i j} \geq 0$ satisfying

$$
\left|a_{h}\left(v_{i}, v_{j}\right)\right| \leq \kappa_{i j} a_{h}\left(v_{i}, v_{i}\right)^{1 / 2} a_{h}\left(v_{j}, v_{j}\right)^{1 / 2}, \quad \forall v_{i} \in N_{h}^{i}, v_{j} \in N_{h}^{i}, i, j=1, \ldots, n .
$$

Then the next lemma can be found in [23]. 
Lemma 3. Assume that the assumptions (A1) and (A2) are satisfied. Then

$$
\begin{aligned}
& \lambda_{\min }(\Pi) \geq \mathcal{C}^{-1}, \\
& \lambda_{\max }(\Pi) \leq \rho(\kappa)+1,
\end{aligned}
$$

where $\rho(\kappa)$ is the spectral radius of $\kappa$.

3.2.2. Convergence results. We now give two examples of the coarse space $N_{h}^{0}$ so that the assumptions (A1) and (A2) are satisfied. Namely, we estimate the two constants $\mathcal{C}$ and $\rho(\kappa)$. For this, let $R_{h}$ be the nodal interpolation operator into $N_{h}$, and let $U_{H}$ be the conforming space of linear polynomials associated with $\mathcal{E}_{H}$. Then, following [17], we define $N_{h}^{0}$ as follows:

$$
N_{h}^{0}=\left\{v \in N_{h}: v=R_{h} \varphi, \varphi \in U_{H}\right\} .
$$

To give the second example, let $\mathcal{E}_{h}$ be the finest triangulation and let $\mathcal{E}_{h}=\mathcal{E}_{H_{J}}$ for some $J \geq 1$ where $\mathcal{E}_{H_{k}}=\mathcal{E}_{k}\left(H_{k}=2^{-k} H, 0 \leq k \leq J\right)$ is constructed by connecting the midpoints of the edges of the triangles in $\mathcal{E}_{k-1}$. Then, following [17], we define the operator $\mathcal{I}_{k-1}^{k}: N_{k-1} \rightarrow N_{k}$ as follows, where $N_{k} \equiv N_{H_{k}}$ is the $P_{1}$ nonconforming space associated with $\mathcal{E}_{k}$ (in particular, $N_{h} \equiv N_{H_{J}}$ ). If $v \in N_{k-1}$ and $E \in \mathcal{E}_{k-1}$ with the vertices $\left(x_{i}, y_{i}\right)$ and the midpoints $\left(\bar{x}_{i}, \bar{y}_{i}\right)$ of its edges, $i=1,2,3$, then

$$
\begin{aligned}
& \mathcal{I}_{k-1}^{k} v\left(\bar{x}_{i}, \bar{y}_{i}\right)=v\left(\bar{x}_{i}, \bar{y}_{i}\right), \quad i=1,2,3, \\
& \mathcal{I}_{k-1}^{k} v\left(x_{i}, y_{i}\right)=\frac{1}{\mathcal{N}_{1}} \sum_{j} v\left(\bar{x}_{j}^{\prime}, \bar{y}_{j}^{\prime}\right) \quad \text { if }\left(x_{i}, y_{i}\right) \notin \partial \Omega, \\
& \mathcal{I}_{k-1}^{k} v\left(x_{i}, y_{i}\right)=\frac{1}{\mathcal{N}_{2}} \sum_{j} v\left(\bar{x}_{j}^{\prime \prime}, \bar{y}_{j}^{\prime \prime}\right) \quad \text { if }\left(x_{i}, y_{i}\right) \in \partial \Omega,
\end{aligned}
$$

where $\mathcal{N}_{1}$ and $\mathcal{N}_{2}$ are the number of the adjacent midpoints $\left(\bar{x}_{j}^{\prime}, \bar{y}_{j}^{\prime}\right)$ and $\left(\bar{x}_{j}^{\prime \prime}, \bar{y}_{j}^{\prime \prime}\right)$ to $\left(x_{i}, y_{i}\right)$ of the edges in $\partial \mathcal{E}_{k-1}$ and the edges on $\partial \Omega$ of the elements in $\mathcal{E}_{k-1}$, respectively. Alternatively, following [37], $\mathcal{I}_{k-1}^{k}: N_{k-1} \rightarrow N_{k}$ can be equivalently defined by

$$
\begin{aligned}
& \mathcal{I}_{k-1}^{k} v\left(\bar{x}_{i}, \bar{y}_{i}\right)=v\left(\bar{x}_{i}, \bar{y}_{i}\right), \quad i=1,2,3, \\
& \mathcal{I}_{k-1}^{k} v\left(x_{i}, y_{i}\right)=\left.\frac{1}{\mathcal{N}_{1}} \sum_{\left(x_{i}, y_{i}\right) \in K_{j}} v\right|_{K_{j}}\left(x_{i}, y_{i}\right) \quad \text { if }\left(x_{i}, y_{i}\right) \notin \partial \Omega, \\
& \mathcal{I}_{k-1}^{k} v\left(x_{i}, y_{i}\right)=\frac{1}{\mathcal{N}_{2}} \sum_{j} v\left(\bar{x}_{j}^{\prime \prime}, \bar{y}_{j}^{\prime \prime}\right) \quad \text { if }\left(x_{i}, y_{i}\right) \in \partial \Omega,
\end{aligned}
$$

where $\mathcal{N}_{1}$ is the number of elements $K_{j} \in \mathcal{E}_{k-1}$ meeting at $\left(x_{i}, y_{i}\right)$ and $\mathcal{N}_{2}$ is defined as in (3.15). Note that (3.15) and (3.16) define the value of $I_{k-1}^{k} v$ at the vertices of elements in $\mathcal{E}_{k}$ and thus can be used to define the continuous piecewise linear function $I_{k-1}^{k} v$ on $\mathcal{E}_{k}$. Hence, $\mathcal{I}_{k-1}^{k} v$ is obviously in $N_{k}$ from its construction $(3.15 \mathrm{c})$ and $(3.16 \mathrm{c})$ on the boundary $\partial \Omega$. It is also a function in $N_{h}$. Now, the second definition of $N_{h}^{0}$ is given by

$$
N_{h}^{0}=\left\{v \in N_{h}: v=\mathcal{I}_{H} \varphi, \varphi \in N_{H}\right\},
$$

where $\mathcal{I}_{H} \equiv \mathcal{I}_{0}^{1}$ and $N_{H}=N_{H_{0}}$. In the context of nonconforming finite elements, the space in (3.17) is a more natural choice for the coarse space $N_{h}^{0}$. Since $U_{H} \subset N_{H}$, the space in (3.14) is a subspace of the space in (3.17). Hence, the following proof applies to both cases. 
Theorem 4. Assume that the additive Schwarz operator $\Pi$ is defined by (3.10) with the coarse space given by (3.14) or (3.17). Then there is a constant independent of $h, H$, and $\delta$ such that the condition number $c(\Pi)$ of $\Pi$ satisfies

$$
c(\Pi) \leq C(1+H / \delta)
$$

It follows from Theorem 4 that if we use a generous overlapping, then the condition number of $\Pi$ is uniformly bounded. The proof of this theorem is given in the next subsection.

3.2.3. Proof of the convergence result. We show (3.18), using a similar result from the conforming elements through an adaptation of Cowsar's arguments [17]. To that end, we need the following two technical lemmas. Below we use the notation

$$
|v|_{k} \equiv|v|_{\mathcal{E}_{k}}=\left(\sum_{E \in \mathcal{E}_{k}}|v|_{H^{1}(E)}^{2}\right)^{1 / 2}, \quad k=0,1, \ldots, J .
$$

Below we use $|v|_{h}=|v|_{\mathcal{E}_{h}}$.

Lemma 5. There are constants $C_{1}$ and $C_{2}$ independent of $h$ and $H$ such that for all $v \in N_{k-1}$, we have

$$
\begin{aligned}
& C_{1}\|v\|_{L^{2}(\Omega)} \leq\left\|\mathcal{I}_{k-1}^{k} v\right\|_{L^{2}(\Omega)} \leq C_{2}\|v\|_{L^{2}(\Omega)}, \\
& C_{1}|v|_{k-1} \leq\left|\mathcal{I}_{k-1}^{k} v\right|_{H^{1}(\Omega)} \leq C_{2}|v|_{k-1} .
\end{aligned}
$$

Proof. The inequality (3.19a) is trivial from the definition of $\mathcal{I}_{k-1}^{k}$. Also, the lower bound in $(3.19 \mathrm{~b})$ is obvious since the degrees of freedom of $N_{k-1}$ are contained in those of the range of the operator $N_{k}$. Thus, it suffices to prove the upper bound in (3.19b). Toward that end, note that for every $v \in N_{k-1},|v|_{k-1}$ is a norm in $N_{k-1}$ equivalent to

$$
\left(\sum_{E \in \mathcal{E}_{k-1}} \sum_{i, j=1}^{3}\left(v\left(\bar{x}_{i}, \bar{y}_{i}\right)-v\left(\bar{x}_{j}, \bar{y}_{j}\right)\right)^{2}\right)^{1 / 2}
$$

where the $\left(\bar{x}_{i}, \bar{y}_{i}\right)$ are the midpoints of the edges of $E$. A similar result holds for every $v \in N_{k}$. Then the upper bound in (3.19b) follows easily from the definition of $\mathcal{I}_{k-1}^{k},(3.20)$, and a simple algebraical computation.

From this lemma we have the corollary.

Corollary 6. There is a constant $C$ independent of $h$ and $H$ such that for $\varphi \in N_{H}$

$$
\begin{aligned}
& \left\|\mathcal{I}_{H} \varphi\right\|_{h} \leq C\|\varphi\|_{\mathcal{E}_{H}}, \\
& \left\|\mathcal{I}_{H} \varphi-\varphi\right\|_{L^{2}(\Omega)} \leq C H\|\varphi\|_{\mathcal{E}_{H}},
\end{aligned}
$$

where we recall that $\mathcal{I}_{H}=\mathcal{I}_{0}^{1}$.

The following lemma was proven in [23] for the conforming finite elements. 
Lemma 7. Let $\mathcal{E}_{h / 2}$ be constructed by connecting the midpoints of the edges of the triangles in $\mathcal{E}_{h}$, and set

$$
U_{h / 2}=\left\{v \in C^{0}(\bar{\Omega}):\left.v\right|_{E} \in P_{1}(E), \forall E \in \mathcal{E}_{h / 2},\left.v\right|_{\partial \Omega}=0\right\}
$$

Then for every $v \in U_{h / 2}$, there is a decomposition $v=\sum_{i=0}^{n} v_{i}$ with $v_{0} \in U_{H}$ and $v_{i} \in U_{h / 2} \cap H_{0}^{1}\left(\Omega_{i}^{\prime}\right)$ such that

$$
\sum_{i=0}^{n}\left|v_{i}\right|_{H^{1}(\Omega)}^{2} \leq C(1+H / \delta)|v|_{H^{1}(\Omega)}^{2},
$$

where $C$ is independent of $h, H$, and $\delta$.

Proof of Theorem 4. Let $N_{h}^{0}$ be given in (3.14). Note that

$$
a_{h}(\Pi v, v)=\sum_{i=0}^{n} a_{h}\left(\Pi_{i} v, v\right) .
$$

Then it follows from Schwarz's inequality and the facts that the $\Pi_{i}$ are projections and the maximum number of the substructures $\Omega_{i}^{\prime}$ that intersect at any point is uniformly bounded that the spectrum of $\Pi$ is bounded above by

$$
1+\max _{(x, y) \in \Omega}\left\{\#\left(i:(x, y) \in \Omega_{i}^{\prime}\right)\right\} .
$$

So we see that the spectrum of $\Pi$ can be obtained without use of the assumption (A2).

Next, let $\mathcal{I}_{h} \equiv \mathcal{I}_{J}^{J+1}: N_{h} \rightarrow U_{h / 2}$ be defined as in (3.15) or in (3.16), and for every $v \in N_{h}$, let $\left(\mathcal{I}_{h} v\right)_{i}$ be the decomposition of $\mathcal{I}_{h} v$ constructed according to Lemma 7. Then we see that $v_{i}=R_{h}\left(\left(\mathcal{I}_{h} v\right)_{i}\right) \in N_{h}^{i}$ and $v=\sum_{i=0}^{n} v_{i}$. Thus, it follows from Lemmas 5 and 7 that

$$
\begin{aligned}
\sum_{i=0}^{n} a_{h}\left(v_{i}, v_{i}\right) & \leq C \sum_{i=0}^{n}\left|R_{h}\left(\left(\mathcal{I}_{h} v\right)_{i}\right)\right|_{h}^{2} \\
& \leq C \sum_{i=0}^{n}\left|\left(\mathcal{I}_{h} v\right)_{i}\right|_{H^{1}(\Omega)}^{2} \\
& \leq C(1+H / \delta)\left|I_{h} v\right|_{H^{1}(\Omega)}^{2} \\
& \leq C(1+H / \delta) a_{h}(v, v)
\end{aligned}
$$

Namely, the assumption (A1) is true, and thus we have the desired result (3.18).

We close this subsection with two remarks. First, a different coarse space from that given in (3.14) and (3.17) was introduced in [37], and the condition number of the resulting additive Schwarz operator $\Pi$ was shown bounded by a constant times $(1+\log (H / h))(1+H / \delta)$. His arguments showed that the constant is independent of jumps in the coefficient $a$ across subdomain interfaces. If the present technique were used to derive (3.18) with $C$ independent of the jumps in the coefficient, the same $\log$ factor would appear in (3.18). Second, while the simple model (2.1) was analyzed, the analysis in this section applies to more general equations, as noted in $[12]$. 
3.3. Two-level multiplicative Schwarz method. We now develop a two-level multiplicative Schwarz algorithm for (3.3).

Two-level multiplicative algorithm. Starting from any initial guess $\psi^{0} \in N_{h}$, we find $\psi^{i} \in N_{h}$ as follows:

(1) Set $v_{-1}=\psi^{i-1}$;

(2) For $j=0,1, \ldots, n$, compute $v_{j}$ by

$$
v_{j}=v_{j-1}+\Pi_{j}\left(\psi_{h}-v_{j-1}\right)
$$

(3) Set $\psi^{i}=v_{n}$.

The computation of $\Pi_{j} \psi_{h}$ in the second step can be easily done through the relation as in (3.12):

$$
a_{h}\left(\Pi_{j} \psi_{h}, w\right)=\left(f_{h}, w\right), \quad \forall w \in N_{h}^{j},
$$

by (3.5) and (3.9). Note that the error $e_{i}=\psi_{h}-\psi^{i}$ satisfies $e_{i+1}=Q e_{i}$, where

$$
Q=\left(I-\Pi_{n}\right)\left(I-\Pi_{n-1}\right) \cdots\left(I-\Pi_{0}\right) .
$$

Thus, the convergence of the multiplicative algorithm is measured from the norm estimate of $Q$. The following abstract theory about the convergence of this multiplicative algorithm is a refinement of a result given in [3].

Lemma 8. Assume that the assumptions (A1) and (A2) are satisfied. Then

$$
\|Q\|_{a} \leq \sqrt{1-\frac{1}{\left(2 \rho(\kappa)^{2}+1\right) \mathcal{C}}}
$$

where the operator norm $\|\cdot\|_{a}$ is measured in the $a_{h}(\cdot, \cdot)$-inner product.

Applying this lemma and the same ideas as in the previous section, we have the next result.

Theorem 9. Assume that the coarse space $N_{h}^{0}$ is defined by (3.14) or by (3.17). Then there is a constant $C$ independent of $h, H$, and $\delta$ such that

$$
\|Q\|_{a} \leq \sqrt{1-\frac{\delta}{C(\delta+H)}} .
$$

3.4. Multilevel Schwarz methods. In this subsection we extend the previous two-level additive and multiplicative Schwarz methods to the corresponding multilevel methods.

Let $\mathcal{E}_{H}=\mathcal{E}_{H_{0}}$ be given and the family $\left\{\mathcal{E}_{H_{k}}\right\}_{k \geq 1}$ be constructed as before. Let $\mathcal{E}_{h}=\mathcal{E}_{H_{J}}$ be the finest triangulation of $\Omega$, i.e., $h=2^{-J} H$. Again, $N_{H_{k}}=N_{k}$ denotes the $P_{1}$ nonconforming finite element space of level $k$ associated with the triangulation $\mathcal{E}_{k}$. Define $(\cdot, \cdot)_{k}$ on $N_{k}$ by

$$
(v, w)_{k}=H_{k}^{2} \sum_{\left(\bar{x}_{i}, \bar{y}_{i}\right) \in \mathcal{M}_{k}} v\left(\bar{x}_{i}, \bar{y}_{i}\right) w\left(\bar{x}_{i}, \bar{y}_{i}\right), \quad v, w \in N_{k}, k=0,1, \ldots, J,
$$


where $\mathcal{M}_{k}$ indicates the set of midpoints of edges in $\partial \mathcal{E}_{k}$. We now introduce several operators. Let $A_{k}: N_{k} \rightarrow N_{k}$ be given by

$$
\left(A_{k} v, w\right)_{k}=a_{k}(v, w), \quad \forall w \in N_{k},
$$

where $a_{k}(\cdot, \cdot)=a_{H_{k}}(\cdot, \cdot)$. As mentioned before, the operator $\mathcal{I}_{k-1}^{k}: N_{k-1} \rightarrow N_{k}$ as defined in (3.15) or (3.16) has the property that $\mathcal{I}_{k-1}^{k} v$ is a continuous piecewise linear function on $\mathcal{E}_{k}$ for $v \in N_{k-1}$, so in fact $\mathcal{I}_{k-1}^{k} v \in N_{h}$. Hence, let $\mathcal{I}_{k} \equiv \mathcal{I}_{k}^{k+1}$ : $N_{k} \rightarrow N_{h}, k=0,1, \ldots, J-1$. Also, define $\mathcal{I}^{k}: N_{h} \rightarrow N_{k}$ and $\tilde{\mathcal{I}}^{k}: N_{h} \rightarrow N_{k}$ by

$$
\begin{array}{ll}
a_{k}\left(\mathcal{I}^{k} v, w\right)=a_{h}\left(v, \mathcal{I}_{k} w\right), & \forall w \in N_{k}, \\
\left(\tilde{\mathcal{I}}^{k} v, w\right)_{k}=\left(v, \mathcal{I}_{k} w\right)_{J}, & \forall w \in N_{k} .
\end{array}
$$

Finally, let $\Lambda_{k}: N_{k} \rightarrow N_{k}$ be a symmetric and positive definite operator with respect to the $(\cdot, \cdot)_{k}$-inner product. Assume that there are constants $\gamma_{0}$ and $\gamma_{1}$ independent of $k$ such that

$$
\gamma_{0}(v, v)_{k} \leq\left(\Lambda_{k} v, v\right)_{k} \leq \gamma_{1}(v, v)_{k}, \quad \forall v \in N_{k}
$$

The operator $\Lambda_{k}$ should be more easily inverted than $A_{k}$; the identity operator on $N_{k}$ is of practical interest among many choices of $\Lambda_{k}$. From these operators we define $S_{k}$ by

$$
\begin{array}{rlrl}
S_{k}^{\prime} & =\mathcal{I}_{k} \Lambda_{k}^{-1} A_{k} \mathcal{I}^{k}, & k=0,1, \ldots, J \\
S_{k} & =\mathcal{C}_{1} H_{k}^{2} S_{k}^{\prime}, & k & =0,1, \ldots, J,
\end{array}
$$

where we assume that $\mathcal{I}_{J}=\mathcal{I}^{J}$ is the identity operator on $N_{h}$, and $\mathcal{C}_{1}$ satisfies

$$
0<s_{k} \leq\left(\mathcal{C}_{1} H_{k}^{2}\right)^{-1}
$$

where $s_{k}$ is the largest eigenvalue of $S_{k}^{\prime}$. It was shown [39] that there is a constant $\mathcal{C}_{1}$ independent of $k$ such that this inequality is indeed satisfied. So the operator $S_{k}$ is well defined. We are now ready to define the multilevel algorithms for (3.5) and thus for (3.3).

Multilevel multiplicative algorithm. Starting from any initial guess $\psi^{0} \in N_{h}$, we find $\psi^{i} \in N_{h}$ as follows:

(1) Set $v_{-1}=\psi^{i-1}$;

(2) For $k=0,1, \ldots, J$, compute $v_{k}$ by

$$
v_{k}=v_{k-1}+S_{k}\left(\psi_{h}-v_{k-1}\right)
$$

(3) Set $\psi^{i}=v_{J}$.

Multilevel additive algorithm. Find $\psi_{h} \in N_{h}$ such that

$$
S \psi_{h} \equiv \sum_{k=0}^{J} S_{k} \psi_{h}=\widehat{f}_{h},
$$

where $\widehat{f}_{h}=\sum_{k=0}^{J} S_{k} \psi_{h}$. 
As remarked in the last two subsections, $S_{k} \psi_{h}$ can be easily obtained from the right-hand side function $f$ thanks to the relation

$$
A_{k} \mathcal{I}^{k}=\tilde{\mathcal{I}}^{k} A_{J}
$$

The following theorem states a convergence result for the above multilevel additive and multiplicative algorithms, which can be obtained from an application of the abstract theory $[3,4,23]$ of multilevel algorithms to the present situation, as shown in [39]. Set

$$
Q=\left(I-S_{J}\right)\left(I-S_{J-1}\right) \cdots\left(I-S_{0}\right) .
$$

Theorem 10. There are constants $C_{0}, C$, and $\tilde{\delta} \in(0,1)$ independent of $h$ and $H$ such that the condition number $c(S)$ of $S$ and the norm $\|Q\|_{a}$ of $Q$ are bounded as follows:

$$
\begin{aligned}
c(S) & \leq \frac{C(1+\tilde{\delta})}{C_{0}(1-\tilde{\delta})}, \\
\|Q\|_{a} & \leq \sqrt{1-\frac{C(1-\tilde{\delta})^{2}}{\left(1-\tilde{\delta}+C_{0} \tilde{\delta}\right)^{2}}} .
\end{aligned}
$$

\section{Rectangular case}

In this section we consider the lowest-order Raviart-Thomas space over rectangles [36] (or equivalently the lowest-order Brezzi-Douglas-Fortin-Marini space [7]).

4.1. Linear system of algebraic equations. Let $\mathcal{E}_{h}$ be a family of quasi-regular partitions of $\Omega$ into rectangles oriented along the coordinate axes, and let $Q_{i, j}(E)$ be the space of polynomials of degree not larger than $i$ in $x$ and $j$ in $y$ on $E$. The rectangular mixed space [36] is defined by

$$
\begin{aligned}
& V_{h}(E)=Q_{1,0}(E) \times Q_{0,1}(E), \\
& W_{h}(E)=P_{0}(E), \\
& L_{h}(e)=P_{0}(e) .
\end{aligned}
$$

For each $E \in \mathcal{E}_{h}$, let $\Delta x_{E}$ and $\Delta y_{E}$ denote the $x$-length and the $y$-length of $E$, respectively, $R_{E}=\Delta x_{E}^{2}+\Delta y_{E}^{2}$, and let $\left(\bar{x}_{E}, \bar{y}_{E}\right)$ denote the center of the rectangle $E$. Let $f_{h}$ be defined as before, and define $J_{h}^{f}$ such that for each $E \in \mathcal{E}_{h},\left.J_{h}^{f}\right|_{E}=$ $f_{E}\left(\Delta y_{E}^{2} x, \Delta x_{E}^{2} y\right) / R_{E}$. For expositional simplicity, let $\mathcal{B}_{h}=\alpha$ be a scalar. Then we again have the next lemma [12]. We emphasize that a similar result holds for a tensor coefficient; see [12] for more information.

Lemma 11. Let

$$
\begin{aligned}
M_{h}(\chi, \mu)= & \sum_{E \in \mathcal{E}_{h}} \frac{1}{(\alpha, 1)_{E}}\left(\chi, \nu_{E}\right)_{\partial E} \cdot\left(\mu, \nu_{E}\right)_{\partial E} \\
& +\sum_{E \in \mathcal{E}_{h}} \frac{12}{(\alpha, 1)_{E} R_{E}}\left(\left(\chi(x, y), \tilde{\nu}_{E}\right)_{\partial E}-\left(\bar{x}_{E}, \bar{y}_{E}\right) \cdot\left(\chi, \tilde{\nu}_{E}\right)_{\partial E}\right) \\
& \times\left(\left(\mu(x, y), \tilde{\nu}_{E}\right)_{\partial E}-\left(\bar{x}_{E}, \bar{y}_{E}\right) \cdot\left(\mu, \tilde{\nu}_{E}\right)_{\partial E}\right), \quad \chi, \mu \in L_{h} \\
F_{h}(\mu)=- & \sum_{E \in \mathcal{E}_{h}} \frac{1}{|E|}\left(J^{f}, 1\right)_{E} \cdot\left(\mu, \nu_{E}\right)_{\partial E}+\sum_{E \in \mathcal{E}_{h}}\left(\mu J^{f}, \nu_{E}\right)_{\partial E}, \quad \mu \in L_{h}
\end{aligned}
$$


where $\tilde{\nu}_{E}^{i}=\left(\nu_{E}^{i(1)},-\nu_{E}^{i(2)}\right)$. Then $\lambda_{h} \in \mathcal{L}_{h}$ satisfies

$$
M_{h}\left(\lambda_{h}, \mu\right)=F_{h}(\mu), \quad \forall \mu \in \mathcal{L}_{h} .
$$

Let the basis in $L_{h}$ be chosen again as usual, and for each $E \in \mathcal{E}_{h}$, set $\left|\nu_{E}^{i}\right|^{\prime}=$ $\left|\nu_{E}^{i(1)}\right|-\left|\nu_{E}^{i(2)}\right|$. Then it follows from (4.1) that the contributions of each rectangle $E$ to the stiffness matrix and the right-hand side are

$$
\begin{aligned}
& m_{i j}^{E}=\frac{1}{(\alpha, 1)_{E}} \bar{\nu}_{E}^{i} \cdot \bar{\nu}_{E}^{j}+\frac{3|E|^{2}}{R_{E}(\alpha, 1)_{E}}\left|\nu_{E}^{i}\right|^{\prime}\left|\nu_{E}^{j}\right|^{\prime}, \\
& F_{i}^{E}=-\frac{\left(J_{h}^{f}, \bar{\nu}_{E}^{i}\right)_{E}}{|E|}+\left(J_{h}^{f}, \nu_{E}^{i}\right)_{e_{E}^{i}} .
\end{aligned}
$$

Namely, we have the linear system for $\lambda_{h}$ :

$$
M \lambda=F .
$$

Lemma 12. Let

(4.4) $N_{h}=\left\{\xi:\left.\xi\right|_{E}=a_{E}^{1}+a_{E}^{2} x+a_{E}^{3} y+a_{E}^{4}\left(x^{2}-y^{2}\right), a_{E}^{i} \in \mathbb{R}, \forall E \in \mathcal{E}_{h}\right.$;

$$
\begin{aligned}
& \text { if } E_{1} \text { and } E_{2} \text { share an edge e, then }\left.\int_{e} \xi\right|_{\partial E_{1}} d s=\left.\int_{e} \xi\right|_{\partial E_{2}} d s \\
& \text { and } \left.\left.\int_{\partial E \cap \partial \Omega} \xi\right|_{\partial \Omega} d s=0\right\} \text {. }
\end{aligned}
$$

Then (4.3) corresponds to the linear system generated by the problem: Find $\psi_{h} \in N_{h}$ such that

$$
a_{h}\left(\psi_{h}, \varphi\right)=\left(f_{h}, \varphi\right), \quad \forall \varphi \in N_{h} .
$$

The equivalence in Lemma 12 is used again to develop the domain decomposition algorithm for (4.3).

After the computation of $\lambda_{h}$, we can calculate $\sigma_{h}$ and $u_{h}$ from (2.4) if they are needed. Setting $\left.\sigma_{h}\right|_{E}=\left(a_{E}+b_{E} x, c_{E}+d_{E} y\right)$, we find [12] that

$$
\begin{aligned}
& a_{E}=\left.\frac{|E|}{(\alpha, 1)_{E}} \sum_{i=1}^{4}\left\{\frac{6 \bar{x}_{E}}{R_{E}}\left(\left|\nu_{E}^{i(1)}\right|-\left|\nu_{E}^{i(2)}\right|\right)-\frac{1}{\Delta x_{E}} \nu_{E}^{i(1)}\right\} \lambda_{h}\right|_{e_{E}^{i}}-\frac{\bar{x}_{E} \Delta y_{E}^{2} f_{E}}{R_{E}}, \\
& b_{E}=\frac{6|E|}{(\alpha, 1)_{E} R_{E}} \sum_{i=1}^{4}\left(-\left|\nu_{E}^{i(1)}\right|+\left|\nu_{E}^{i(2)}\right|\right)+\frac{\Delta y_{E}^{2} f_{E}}{R_{E}}, \\
& c_{E}=\left.\frac{|E|}{(\alpha, 1)_{E}} \sum_{i=1}^{4}\left\{\frac{6 \bar{y}_{E}}{R_{E}}\left(-\left|\nu_{E}^{i(1)}\right|+\left|\nu_{E}^{i(2)}\right|\right)-\frac{1}{\Delta y_{E}} \nu_{E}^{i(2)}\right\} \lambda_{h}\right|_{e_{E}^{i}}-\frac{\bar{y}_{E} \Delta x_{E}^{2} f_{E}}{R_{E}}, \\
& d_{E}=\frac{6|E|}{(\alpha, 1)_{E} R_{E}} \sum_{i=1}^{4}\left(\left|\nu_{E}^{i(1)}\right|-\left|\nu_{E}^{i(2)}\right|\right)+\frac{\Delta x_{E}^{2} f_{E}}{R_{E}} .
\end{aligned}
$$

Also, for each $E$ in $\mathcal{E}_{h}$,

$$
\left.u_{h}\right|_{E}=\left.\frac{1}{2 R_{E}} \sum_{i=1}^{4}\left(\Delta y_{E}^{2}\left|\nu_{E}^{i(1)}\right|+\Delta x_{E}^{2}\left|\nu_{E}^{i(2)}\right|\right) \lambda_{h}\right|_{e_{E}^{i}}+\frac{(\alpha, 1)_{E}|E| f_{E}}{12 R_{E}} .
$$

We remark that the matrix $M$ in (4.4) has at most seven nonzero entries per row. It is symmetric and positive definite. However, in general, it is not an $M$-matrix. 
4.2. Two-level additive Schwarz method. Let $\mathcal{E}_{H}$ be a quasi-regular coarse triangulation of $\Omega$ into nonoverlapping rectangular substructures $\Omega_{i}, i=1, \ldots, n$, and let $\mathcal{E}_{h}$ be a quasi-regular refinement of $\mathcal{E}_{H}$ into rectangles. Again, let $\left\{\Omega_{i}^{\prime}\right\}$ be an overlapping domain decomposition of $\Omega$ which aligns with the boundary $\partial \Omega$. The overlap parameter $\delta$ is defined as before. Associated with each $\Omega_{i}^{\prime}$, let $N_{h}^{i}$ be the restriction of the nonconforming finite element $N_{h}$ to $\Omega_{i}^{\prime}$. With these, the form of the additive Schwarz method given in (3.11) and (3.12) remains the same. Moreover, a parallel analysis could be given here. However, we here show how to use the established results of the triangular elements to analyze the rectangular case.

Let $\widehat{\mathcal{E}}_{h}$ be the triangulation of $\Omega$ into triangles obtained by connecting the two opposite vertices of the rectangles in $\mathcal{E}_{h}$, as illustrated in Figure 1. Associated with $\widehat{\mathcal{E}}_{h}$, let $\widehat{N}_{h}$ be the $P_{1}$ nonconforming finite element space as defined in (3.4). Then we define the operator $\widehat{\mathcal{I}}_{h}: N_{h} \rightarrow \widehat{N}_{h}$ as follows. If $v \in N_{h}$ and $e$ is an edge of a triangle in $\widehat{N}_{h}$, then $\widehat{\mathcal{I}}_{h} v \in \widehat{N}_{h}$ is defined by

$$
\frac{1}{|e|}\left(\widehat{\mathcal{I}}_{h} v, 1\right)_{e}=\frac{1}{|e|}(v, 1)_{e} .
$$

Lemma 13. There is a constant $C$ independent of $h$ such that for all $v \in N_{h}$

$$
\begin{aligned}
& \left\|\widehat{\mathcal{I}}_{h} v\right\|_{L^{2}(\Omega)} \leq C\|v\|_{L^{2}(\Omega)}, \\
& \left\|\widehat{\mathcal{I}}_{h} v\right\|_{h} \leq C\|v\|_{h} .
\end{aligned}
$$

Proof. We first prove the inequality (4.7a). From (4.6) it follows that

$$
\begin{aligned}
\left\|\widehat{\mathcal{I}}_{h} v\right\|_{L^{2}(\Omega)}^{2} & =\sum_{E \in \mathcal{E}_{h}}\left\|\widehat{\mathcal{I}}_{h} v\right\|_{L^{2}(E)}^{2} \\
& \leq C \sum_{E \in \mathcal{E}_{h}} \sum_{i=1}^{4}\left(\int_{e_{E}^{i}}\left|\widehat{\mathcal{I}}_{h} v\right| d e\right)^{2} \\
& \leq C \sum_{E \in \widehat{\mathcal{E}}_{h}} \sum_{i=1}^{3}\left(\int_{e_{E}^{i}}|v| d e\right)^{2} \\
& \leq C\|v\|_{L^{2}(\Omega)}^{2},
\end{aligned}
$$

from which (4.7a) follows.

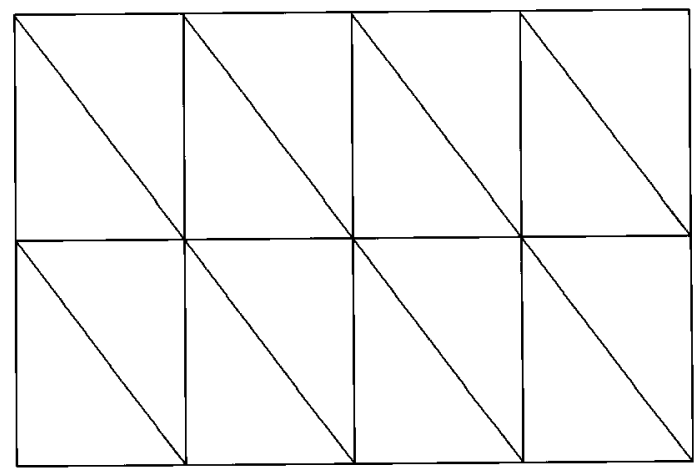

Figure 1. A triangular refinement of rectangles 
We now prove the second inequality, which follows from the first one. Given $v \in N_{h}$, define $\xi \in N_{h}, w \in \widehat{N}_{h}$, and $z \in H_{0}^{1}(\Omega)$ by

$$
\begin{aligned}
& a_{h}(v, \zeta)=(\xi, \zeta), \quad \forall \zeta \in N_{h}, \\
& a_{h}(w, \zeta)=(\xi, \zeta), \quad \forall \zeta \in \widehat{N}_{h}, \\
& a_{h}(z, \zeta)=(\xi, \zeta), \quad \forall \zeta \in H_{0}^{1}(\Omega) .
\end{aligned}
$$

Note that $\|z\|_{H^{2}(\Omega)} \leq C\|\xi\|_{L^{2}(\Omega)}$ by elliptic regularity, and that $v$ and $w$ are approximations to $z$ with the usual error estimates [1]. Thus, it follows from an inverse inequality and (4.7a) that

$$
\begin{aligned}
\left\|\widehat{\mathcal{I}}_{h} v\right\|_{h} & \leq\left\|\widehat{\mathcal{I}}_{h}(v-w)\right\|_{h}+\|w\|_{h} \\
& \leq C\left(h^{-1}\left\|\widehat{\mathcal{I}}_{h}(v-w)\right\|_{L^{2}(\Omega)}+\|v-w\|_{h}+\|v\|_{h}\right) \\
& \leq C\left(h^{-1}\|v-w\|_{L^{2}(\Omega)}+\|v\|_{h}\right) \\
& \leq C\left(h^{-1}\left(\|v-z\|_{L^{2}(\Omega)}+\|w-z\|_{L^{2}(\Omega)}\right)+\|v\|_{h}\right) \\
& \leq C\left(h\|\xi\|_{L^{2}(\Omega)}+\|v\|_{h}\right) .
\end{aligned}
$$

Finally, by (4.8), we see that

$$
\|\xi\|_{L^{2}(\Omega)}^{2}=a_{h}(v, \xi) \leq C\|v\|_{h}\|\xi\|_{h} \leq C h^{-1}\|v\|_{h}\|\xi\|_{L^{2}(\Omega)}
$$

and $(4.7 \mathrm{~b})$ follows.

Let $R_{h}$ be the interpolation operator into $N_{h}$, and define the coarse space $N_{h}^{0}$ by

$$
N_{h}^{0}=\left\{v \in N_{h}: v=R_{h} \varphi, \varphi \in \widehat{N}_{h}^{0}\right\},
$$

where $\widehat{N}_{h}^{0}$ is a triangular coarse space such that $R_{h} \varphi$ is well defined for every $\varphi \in \widehat{N}_{h}^{0}$.

Theorem 14. Assume that $\widehat{N}_{h}^{0}$ is such a triangular coarse space that the result in Theorem 4 is true and that the rectangular coarse space $N_{h}^{0}$ is given by (4.9). Then the condition number $c(\Pi)$ of the additive Schwarz operator $\Pi$ in the rectangular case satisfies

$$
c(\Pi) \leq C(1+H / \delta),
$$

where $C$ is independent of $h, H$, and $\delta$.

Proof. The spectrum of $\Pi$ can be bounded as before. It again suffices to prove the assumption (A1). For every $v \in N_{h}$, let $\left(\widehat{\mathcal{I}}_{h} v\right)_{i} \in \widehat{N}_{h}^{i}$ be the decomposition of $\widehat{\mathcal{I}}_{h} v \in \widehat{N}_{h}$ constructed from the triangular case. Let $v_{i}=R_{h}\left(\left(\widehat{\mathcal{I}}_{h} v\right)_{i}\right) \in N_{h}^{i}$. Then we see that $v=\sum_{i=0}^{n} v_{i}$. Thus, by Theorem 4 and Lemma 13, we obtain

$$
\begin{aligned}
\sum_{i=0}^{n} a_{h}\left(v_{i}, v_{i}\right) & \leq C \sum_{i=0}^{n} a_{h}\left(\left(\widehat{\mathcal{I}}_{h} v\right)_{i},\left(\widehat{\mathcal{I}}_{h} v\right)_{i}\right) \\
& \leq C(1+H / \delta) a_{h}\left(\widehat{\mathcal{I}}_{h} v, \widehat{\mathcal{I}}_{h} v\right) \\
& \leq C(1+H / \delta) a_{h}(v, v),
\end{aligned}
$$

and (A1) follows. 
Let $\mathcal{E}_{h}=\mathcal{E}_{H_{J}}$ for some $J \geq 1$ where $\mathcal{E}_{H_{k}}=\mathcal{E}_{k}\left(H_{k}=2^{-k} H, 0 \leq k \leq J\right)$ is constructed by connecting the midpoints of the edges of the rectangles in $\mathcal{E}_{k-1}$. For each $0 \leq k \leq J$, let $\widehat{\mathcal{E}}_{k}$ be the triangulation of $\Omega$ into triangles corresponding to $\mathcal{E}_{h}$. Associated with each $\widehat{\mathcal{E}}_{k}$, let $\widehat{N}_{k}$ be the $P_{1}$ nonconforming finite element space as defined in (3.4). Then it is easy to see that $N_{h}^{0}$ can be constructed from $\widehat{N}_{h}^{0}$ by means of (3.14) or (3.17).

The same idea also applies to the analysis of the two-level multiplicative algorithm, and the same result given in Theorem 11 remains valid here.

4.3. Multilevel Schwarz methods. Let $\mathcal{E}_{H}=\mathcal{E}_{H_{0}}$ be given and the family $\left\{\mathcal{E}_{H_{k}}\right\}_{k \geq 1}$ be constructed as above. Let $\mathcal{E}_{h}=\mathcal{E}_{H_{J}}$ be the finest triangulation of $\Omega$, i.e., $h=2^{-J} H$, for some $J \geq 1$, and let $N_{H_{k}}=N_{k}$ denote the nonconforming finite element space of level $k$ associated with the triangulation $\mathcal{E}_{k}$, as defined in (4.4). For each $k$, we introduce the continuous bilinear functions

$$
U_{k}=\left\{\xi \in C^{0}(\bar{\Omega}):\left.\xi\right|_{E} \in Q_{1,1}(E), \forall E \in \mathcal{E}_{k} \text { and }\left.\xi\right|_{\partial \Omega}=0\right\}
$$

Unlike the triangular case, $U_{k} \not \subset N_{k}$. Thus, the intergrid transfer operator $\mathcal{I}_{k-1}^{k}$ : $N_{k-1} \rightarrow N_{k}$ cannot be defined as in (3.15) and (3.16). Hence, the convergence analysis in $\S 3.4$ does not apply here. Fortunately, we can use the idea of the proof in Theorem 14 to construct the operator $\mathcal{I}_{k-1}^{k}$.

For each $k$, let $\widehat{\mathcal{E}}_{k}$ be the triangulation of $\Omega$ into triangles obtained from $\mathcal{E}_{k}$ using the above manner (see Figure 1), and let $\widehat{\mathcal{I}}_{k}: N_{k} \rightarrow \widehat{N}_{k}$ be defined as in (4.6). Let $\overline{\mathcal{I}}_{k}: \widehat{N}_{k} \rightarrow \widehat{N}_{h}$ be defined as in (3.15) or (3.16). Then we define $\mathcal{I}_{k}: N_{k} \rightarrow N_{h}$ by

$$
\mathcal{I}_{k}=R_{h} \overline{\mathcal{I}}_{k} \widehat{\mathcal{I}}_{k}
$$

Define $(\cdot, \cdot)_{k}$ on $N_{k}$ by

$$
(v, w)_{k}=\sum_{e \in \partial \mathcal{E}_{k}} H_{k}(v, w)_{e}
$$

We can now introduce the operators $A_{k}, \mathcal{I}^{k}, \tilde{\mathcal{I}}^{k}, \Lambda_{k}$, and $S_{k}$ as before. Namely, $A_{k}: N_{k} \rightarrow N_{k}$ is given by

$$
\left(A_{k} v, w\right)_{k}=a_{k}(v, w), \quad \forall w \in N_{k},
$$

$\mathcal{I}^{k}: N_{h} \rightarrow N_{k}$ and $\tilde{\mathcal{I}}^{k}: N_{h} \rightarrow N_{k}$ are given by

$$
\begin{array}{ll}
a_{k}\left(\mathcal{I}^{k} v, w\right)=a_{h}\left(v, \mathcal{I}_{k} w\right), & \forall w \in N_{k}, \\
\left(\tilde{\mathcal{I}}^{k} v, w\right)_{k}=\left(v, \mathcal{I}_{k} w\right)_{J}, & \forall w \in N_{k},
\end{array}
$$

and $\Lambda_{k}: N_{k} \rightarrow N_{k}$ is a symmetric and positive definite operator with respect to the $(\cdot, \cdot)_{k}$-inner product such that there are constants $\gamma_{0}$ and $\gamma_{1}$ independent of $k$ satisfying

$$
\gamma_{0}(v, v)_{k} \leq\left(\Lambda_{k} v, v\right)_{k} \leq \gamma_{1}(v, v)_{k}, \quad \forall v \in N_{k}
$$


From these operators we define $S_{k}$ by

$$
\begin{array}{ll}
S_{k}^{\prime}=\mathcal{I}_{k} \Lambda_{k}^{-1} A_{k} \mathcal{I}^{k}, & k=0,1, \ldots, J, \\
S_{k}=\mathcal{C}_{1} H_{k}^{2} S_{k}^{\prime}, & k=0,1, \ldots, J,
\end{array}
$$

where $\mathcal{C}_{1}$ satisfies an inequality similar to $(3.25)$. With the operators $S_{k}$, the multilevel additive and multiplicative Schwarz algorithms can be defined as in $\S 3.4$, and the convergence results directly follow from those in Theorem 10 by means of Lemma 13.

\section{SimpleXES}

Let now $\mathcal{E}_{h}$ be a partition of $\Omega$ into simplexes. The lowest-order Raviart-ThomasNedelec space $[36,34]$ defined over $\mathcal{E}_{h}$ is given by

$$
\begin{aligned}
& V_{h}(E)=\left(P_{0}(E)\right)^{3} \oplus\left((x, y, z) P_{0}(E)\right), \\
& W_{h}(E)=P_{0}(E), \\
& L_{h}(e)=P_{0}(e) .
\end{aligned}
$$

In the present case the results in Lemmas 1 and 2 remain the same if we define the nonconforming finite element space

$$
N_{h}=\left\{v \in L^{2}(\Omega):\left.v\right|_{E} \in P_{1}(E), \forall E \in \mathcal{E}_{h} ; v\right. \text { is continuous at the barycenters }
$$
of interior faces and vanishes at the barycenters of faces on $\partial \Omega\}$.

Moreover, for each simplex $E \in E_{h}$, its contributions to the stiffness matrix and the right-hand side are

$$
m_{i j}^{E}=\bar{\nu}_{E}^{i} \beta^{E} \bar{\nu}_{E}^{j}, \quad F_{i}^{E}=-\frac{\left(J_{h}^{f}, \bar{\nu}_{E}^{i}\right)_{E}}{|E|}+\left(J_{h}^{f}, \nu_{E}^{i}\right)_{e_{E}^{i}},
$$

where $J_{h}^{f}=f_{h}(x, y, z) / 3$. For each $E \in \mathcal{E}_{h}$, let $\left.\sigma_{h}\right|_{E}=\left(a_{E}^{1}+b_{E} x, a_{E}^{2}+b_{E} y, a_{E}^{3}+\right.$ $\left.b_{E} z\right)$. Then $\sigma_{h}$ and $u_{h}$ are computed from the following relations:

$$
\begin{aligned}
b_{E}= & \frac{f_{E}}{3}, \\
a_{E}^{j}= & -\left.\sum_{i=1}^{4}\left|e_{E}^{i}\right|\left(\beta_{j 1}^{E} \nu_{E}^{i(1)}+\beta_{j 2}^{E} \nu_{E}^{i(2)}+\beta_{j 3}^{E} \nu_{E}^{i(3)}\right) \lambda_{h}\right|_{e_{E}^{i}} \\
& -\frac{f_{E}}{3} \sum_{i=1}^{3}\left(\beta_{j i}^{E}\left(\alpha_{i 1} x+\alpha_{i 2} y+\alpha_{i 3} z\right), 1\right)_{E}, \quad j=1,2,3, \\
u_{E}= & \frac{1}{3|E|}\left(\left(\mathcal{B}_{h} \sigma_{h},(x, y, z)\right)_{E}+\left.\sum_{i=1}^{4} \lambda_{h}\right|_{e_{E}^{i}}\left((x, y, z), \nu_{E}^{i}\right)_{e_{E}^{i}}\right) .
\end{aligned}
$$

The two-level Schwarz method can be defined as in $\S 3$. If $\mathcal{E}_{H_{0}}$ is given and each $\mathcal{E}_{H_{k+1}}$ is a regular refinement of $\mathcal{E}_{H_{k}}$ into eight times as many elements by joining the barycenters of the faces of the elements in $\mathcal{E}_{H_{k}}$, then the definition of the multilevel Schwarz method remains unchanged provided that the intergrid transfer operator $\mathcal{I}_{k-1}^{k}: N_{k-1} \rightarrow N_{k}$ is given as in (3.15) or (3.16). 


\section{Rectangular parallelepipeds}

Let now $\mathcal{E}_{h}$ be a decomposition of $\Omega$ into rectangular parallelepipeds oriented along the coordinate axes. The lowest-order Raviart-Thomas-Nedelec space [34] defined over $\mathcal{E}_{h}$ (equivalently, the lowest-order Brezzi-Douglas-Fortin-Marini space [7]) is given by

$$
\begin{aligned}
& V_{h}(E)=Q_{1,0,0}(E) \times Q_{0,1,0}(E) \times Q_{0,0,1}(E), \\
& W_{h}(E)=P_{0}(E), \\
& L_{h}(e)=P_{0}(e) .
\end{aligned}
$$

In this case the nonconforming space $N_{h}$ is given by

$$
\begin{gathered}
N_{h}=\left\{\xi:\left.\xi\right|_{E}=a_{E}^{1}+a_{E}^{2} x+a_{E}^{3} y+a_{E}^{4} z+a_{E}^{5}\left(x^{2}-y^{2}\right)+a_{E}^{6}\left(x^{2}-z^{2}\right),\right. \\
a_{E}^{i} \in \mathbb{R}, \forall E \in \mathcal{E}_{h} ; \text { if } E_{1} \text { and } E_{2} \text { share a face } e \\
\text { then } \left.\left.\int_{e} \xi\right|_{\partial E_{1}} d s=\left.\int_{e} \xi\right|_{\partial E_{2}} d s ; \text { and }\left.\int_{\partial E \cap \partial \Omega} \xi\right|_{\partial \Omega} d s=0\right\} .
\end{gathered}
$$

Then the results given in Lemmas 13 and 14 can be extended to the present case. For each $E \in \mathcal{E}_{h}$, set

$$
\begin{aligned}
& R_{E}=\frac{1}{\Delta x_{E}^{2}}+\frac{1}{\Delta y_{E}^{2}}+\frac{1}{\Delta z_{E}^{2}}, \\
& \left.J_{h}^{f}\right|_{E}=\frac{f_{E}}{R_{E}}\left(\frac{x}{\Delta x_{E}^{2}}, \frac{y}{\Delta y_{E}^{2}}, \frac{z}{\Delta z_{E}^{2}}\right),
\end{aligned}
$$

and

$$
\begin{aligned}
& \tilde{\nu}_{E}^{i, \prime}=\left(\frac{\left|\nu^{i(1)}\right|}{\Delta x_{E}}, \frac{\left|\nu^{i(2)}\right|}{\Delta y_{E}}, \frac{\left|\nu^{i(3)}\right|}{\Delta z_{E}}\right), \\
& \left|\nu_{E}^{i}\right|^{\prime}=\frac{\left|\nu_{E}^{i(1)}\right|}{\Delta x_{E}^{2}}+\frac{\left|\nu_{E}^{i(2)}\right|}{\Delta y_{E}^{2}}+\frac{\left|\nu_{E}^{i(3)}\right|}{\Delta z_{E}^{2}}
\end{aligned}
$$

Then the contributions of the rectangular parallelepiped $E \in \mathcal{E}_{h}$ to the stiffness matrix and the right-hand side are

$$
\begin{aligned}
& m_{i j}^{E}=\frac{1}{(\alpha, 1)_{E}} \bar{\nu}_{E}^{i} \cdot \bar{\nu}_{E}^{j}+\frac{3|E|^{2}}{(\alpha, 1)_{E}}\left(\tilde{\nu}_{E}^{i, \prime} \cdot \tilde{\nu}_{E}^{j, \prime}-\frac{1}{R_{E}}\left|\nu_{E}^{i}\right|^{\prime}\left|\nu_{E}^{j}\right|^{\prime}\right), \\
& F_{i}^{E}=-\frac{\left(J_{h}^{f}, \bar{\nu}_{E}^{i}\right)_{E}}{|E|}+\left(J_{h}^{f}, \nu_{E}^{i}\right)_{e_{E}^{i}} .
\end{aligned}
$$

For $E \in \mathcal{E}_{h}$, let $\left.\sigma_{h}\right|_{E}=\left(a_{E}+b_{E} x, c_{E}+d_{E} y, s_{E}+t_{E} z\right)$. Then it follows from (2.4) [12] that

$$
\begin{aligned}
a_{E}=-\frac{6 \bar{x}_{E}|E|}{(\alpha, 1)_{E} \Delta x_{E}^{2} R_{E}} \sum_{i=1}^{6}\{ & \left(1-\Delta x_{E}^{2} R_{E}\right) \frac{\left|\nu_{E}^{i(1)}\right|}{\Delta x_{E}^{2}}+\frac{\left|\nu_{E}^{i(2)}\right|}{\Delta y_{E}^{2}} \\
+ & \left.\frac{\left|\nu_{E}^{i(3)}\right|}{\Delta z_{E}^{2}}+\frac{\Delta x_{E} R_{E}}{6 \bar{x}_{E}} \nu_{E}^{i(1)}\right\}\left.\lambda_{h}\right|_{e_{E}^{i}}-\frac{\bar{x}_{E} f_{E}}{\Delta x_{E}^{2} R_{E}}
\end{aligned}
$$




$$
\begin{aligned}
b_{E}=\frac{6|E|}{(\alpha, 1)_{E} \Delta x_{E}^{2} R_{E}} \sum_{i=1}^{6}\left\{\left(1-\Delta x_{E}^{2} R_{E}\right) \frac{\left|\nu_{E}^{i(1)}\right|}{\Delta x_{E}^{2}}\right. & +\frac{\left|\nu_{E}^{i(2)}\right|}{\Delta y_{E}^{2}} \\
& \left.+\frac{\left|\nu_{E}^{i(3)}\right|}{\Delta z_{E}^{2}}\right\}\left.\lambda_{h}\right|_{e_{E}^{i}}+\frac{f_{E}}{\Delta x_{E}^{2} R_{E}} ;
\end{aligned}
$$

similar expressions hold for $c_{E}, d_{E}, s_{E}$, and $t_{E}$. Finally,

$$
u_{E}=\left.\frac{1}{2 R_{E}} \sum_{i=1}^{6}\left\{\frac{\left|\nu_{E}^{i(1)}\right|}{\Delta x_{E}^{2}}+\frac{\left|\nu_{E}^{i(2)}\right|}{\Delta y_{E}^{2}}+\frac{\left|\nu_{E}^{i(3)}\right|}{\Delta z_{E}^{2}}\right\} \lambda_{h}\right|_{e_{E}^{i}}+\frac{f_{E}(\alpha, 1)_{E}}{12 R_{E}|E|} .
$$

The two-level Schwarz method can be defined as in the rectangular case. If $\mathcal{E}_{H_{0}}$ is given and each $\mathcal{E}_{H_{k+1}}$ is a regular refinement of $\mathcal{E}_{H_{k}}$ into eight times as many elements, then the multilevel Schwarz method can also similarly be defined. Moreover, the convergence result follows from that for the simplexes if an appropriate operator can be defined from the nonconforming space on rectangular parallelepipeds to that on simplexes. This can be done as follows.

Let $\widehat{\mathcal{E}}_{h}$ be the triangulation of $\Omega$ into simplexes obtained by dividing each parallelepiped in $\mathcal{E}_{h}$ into six tetrahedra, as illustrated in Figure 2, or into five tetrahedra, as shown in Figure 3. Also, let $\widehat{N}_{h}$ be the corresponding $P_{1}$ nonconforming space as given in the previous section. Then, if $v \in N_{h}$ and $e$ is a face of a tetrahedron in $\widehat{N}_{h}$, we define $\widehat{\mathcal{I}}_{h} v$ by

$$
\frac{1}{|e|}\left(\widehat{\mathcal{I}}_{h} v, 1\right)_{e}=\frac{1}{|e|}(v, 1)_{e} .
$$
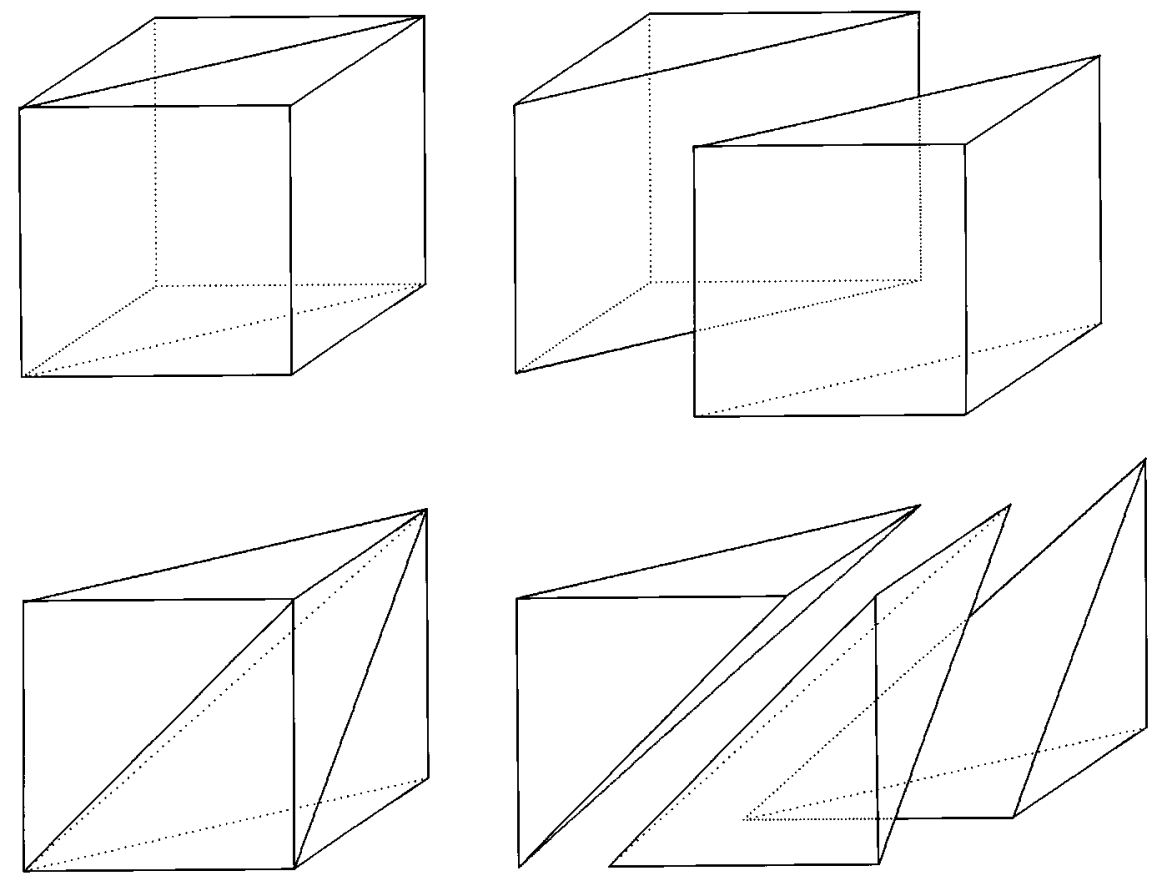

Figure 2. A rectangular parallelepiped divided into six tetrahedra 

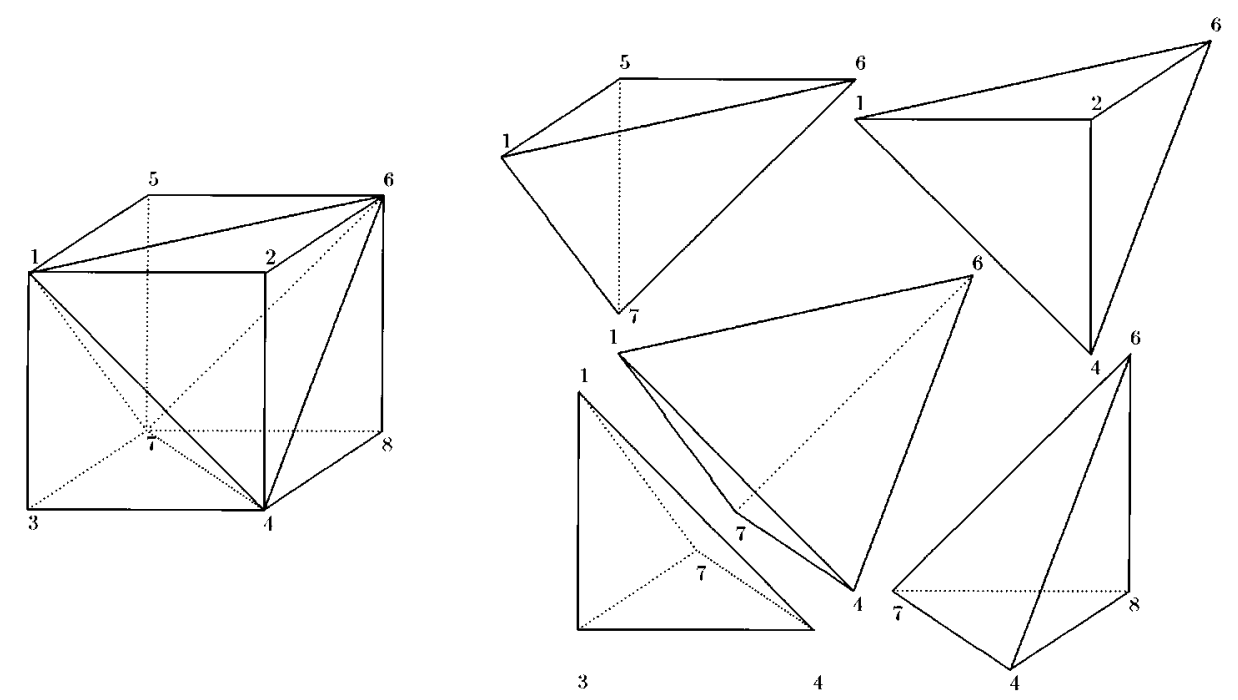

FIGURE 3. A rectangular parallelepiped divided into five tetrahedra

It can be shown as in Lemma 13 that the stability results similar to (4.7) hold for $\widehat{\mathcal{I}}_{h}$. Thus, if the coarse space is given as in (4.9), the convergence result in Theorem 14 remains the same for the rectangular parallelepipeds.

\section{PRismatic ElEments}

Let now $\Omega$ be of the form $\Omega=G \times[0,1]$ with $G \subset \mathbb{R}^{2}$ and $\mathcal{E}_{h}$ be a partition of $\Omega$ into prisms with three vertical edges parallel to the $z$-axis and two horizontal faces in the $(x, y)$-plane. The lowest-order Nedelec space [35] defined over $\mathcal{E}_{h}$ (equivalently, the lowest-order Chen-Douglas space [14]) is given by

$$
\begin{aligned}
& V_{h}(E)=\left(P_{0}(E)\right)^{3} \oplus\left(\left((x, y) P_{0}(E), z P_{0}(E)\right)\right), \\
& W_{h}(E)=P_{0}(E) \\
& L_{h}(e)=P_{0}(e) .
\end{aligned}
$$

The corresponding nonconforming finite element space is given by

$$
\begin{aligned}
N_{h}=\{\xi & :\left.\xi\right|_{E}=a_{E}^{1}+a_{E}^{2} x+a_{E}^{3} y+a_{E}^{4} z+a_{E}^{5}\left(x^{2}+y^{2}-2 z^{2}\right), a_{E}^{i} \in \mathbb{R}, \forall E \in \mathcal{E}_{h} ; \\
& \text { if } E_{1} \text { and } E_{2} \text { share a face } e, \text { then }\left.\int_{e} \xi\right|_{\partial E_{1}} d s=\left.\int_{e} \xi\right|_{\partial E_{2}} d s ; \\
& \text { and } \left.\left.\int_{\partial E \cap \partial \Omega} \xi\right|_{\partial \Omega} d s=0\right\} .
\end{aligned}
$$

Again, the results given in Lemmas 11 and 12 remain the same. Furthermore, for each prism $E \in \mathcal{E}_{h}$, its contributions to the stiffness matrix and the righthand side and the restriction of $\sigma_{h}$ and $u_{h}$ to $E$ can be explicitly determined as in the triangular and rectangular cases; for more details on these expressions for the prismatic elements, refer to [12]. 
The two-level and multilevel Schwarz methods can be defined as before. The convergence result follows from the corresponding result for the simplexes if each prism is divided into three tetrahedra as in Figure 2 and the operator $\widehat{\mathcal{I}}_{h}: N_{h} \rightarrow \widehat{N}_{h}$ is defined as in (6.1). We end this section with a remark that with a linearization approach the problem of solving quasilinear problems reduces to one of solving symmetric linear problems [9], and the theory of the paper applies.

\section{Numerical EXAMPLE}

In this section the two-level additive Schwarz algorithm described in $\S 3$ is applied to the model problem

$$
\begin{array}{ll}
-\Delta u=f & \text { in } \Omega=(0,1)^{3}, \\
u=1 & \text { on } \partial \Omega .
\end{array}
$$

Comparison of numerical experiments among the domain decomposition methods developed in the previous sections will be reported in a forthcoming paper. The right-hand side $f$ is given by

$$
f(x, y, z)=3 \pi^{2} \sin (\pi x) \sin (\pi y) \sin (\pi z),
$$

so that the exact solution is

$$
u(x, y, z)=1+\sin (\pi x) \sin (\pi y) \sin (\pi z) .
$$

The domain $\Omega$ is first divided into uniform cubes, and then each cube is partitioned into five tetrahedra, as shown in Figure 3. The lowest-order Raviart-Thomas space over a uniform decomposition of $\Omega$ into simplexes is exploited here. The conjugate gradient method is exploited with the stopping criterion that the relative residual as measured in the energy norm is less than $10^{-8}$. The experiments in Tables 1 and 2 report the condition number in the cases of the overlap parameter $\delta=H / 4$ and $\delta=h$. In the tables, $n$ is the number of the subdomains, $c(\Pi)$ is the condition number of the two-level additive Schwarz algorithm, and \# is the number of iterations needed to achieve the desired accuracy. From these results we see that the condition number depends linearly on the ratio of the subdomain size to the overlap parameter and is uniformly bounded. Also, the number of iterations is bounded independently of the mesh sizes and the number of decompositions. Hence, the experimental results coincide with the theory established before. An extension of the present approach to other substructuring methods such as those in $[38,29,5]$ will be discussed in a forthcoming paper.

TABLE 1 . The condition number with $\delta=H / 4$

\begin{tabular}{|c|l|l|l|l|l|l|}
\hline $1 / h$ & 16 & 16 & 24 & 24 & 32 & 32 \\
\hline $\mathrm{n}$ & 8 & 64 & 8 & 27 & 8 & 64 \\
\hline$c(\Pi)$ & 5.86 & 5.12 & 6.08 & 6.67 & 6.51 & 6.81 \\
\hline$\#$ & 8 & 9 & 9 & 8 & 8 & 9 \\
\hline
\end{tabular}


TABLE 2. The condition number with $\delta=h$

\begin{tabular}{|c|l|l|l|l|}
\hline $1 / h$ & 36 & 36 & 48 & 48 \\
\hline $\mathrm{n}$ & 8 & 64 & 8 & 64 \\
\hline$c(\Pi)$ & 13.74 & 12.04 & 12.96 & 12.55 \\
\hline$\#$ & 12 & 12 & 13 & 12 \\
\hline
\end{tabular}

\section{ACKNOWLEDGMENTS}

The author wishes to thank the referee for comments leading to an improved presentation of this paper.

\section{REFERENCES}

1. T. Arbogast and Zhangxin Chen, On the implementation of mixed methods as nonconforming methods for second-order elliptic problems, Math. Comp. 64 (1995), 943-972. CMP 95:04

2. D. N. Arnold and F. Brezzi, Mixed and nonconforming finite element methods: implementation, postprocessing and error estimates, RAIRO Modél. Math. Anal. Numér. 19 (1985), 7-32. MR 87g:65126

3. J. H. Bramble, J. E. Pasciak, J. Wang, and J. Xu, Convergence estimates for product iterative methods with applications to domain decomposition, Math. Comp. 57 (1991), 1-21. MR 92d:65094

4. Convergence estimates for multigrid algorithms without regularity assumptions, Math. Comp. 57 (1991), 23-46. MR 91m:65158

5. S. Brenner, Two-level additive Schwarz preconditioners for nonconforming finite element methods, Preprint.

6. F. Brezzi, J. Douglas, Jr., R. Durán, and M. Fortin, Mixed finite elements for second order elliptic problems in three variables, Numer. Math. 51 (1987), 237-250. MR 88f:65190

7. F. Brezzi, J. Douglas, Jr., M. Fortin, and L. D. Marini, Efficient rectangular mixed finite elements in two and three space variables, RAIRO Modél. Math. Anal. Numér. 21 (1987), 581-604. MR 88j:65249

8. F. Brezzi, J. Douglas, Jr., and L. D. Marini, Two families of mixed finite elements for second order elliptic problems, Numer. Math. 47 (1985), 217-235. MR 87g:65133

9. Zhangxin Chen, On the existence, uniqueness and convergence of nonlinear mixed finite element methods, Mat. Apl. Comput. 8 (1989), 241-258. MR 91g:65139

10. Analysis of mixed methods using conforming and nonconforming finite element methods, RAIRO Modél. Math. Anal. Numér. 27 (1993), 9-34. MR 94c:65132

11. _ BDM mixed methods for a nonlinear elliptic problem, J. Comp. Appl. Math. 53 (1994), 207-223. CMP 95:05

12. __ Equivalence between and multigrid algorithms for nonconforming and mixed methods for second order elliptic problems, East-West J. Numer. Math. 4 (1996) (to appear).

13. Zhangxin Chen and J. Douglas, Jr., Approximation of coefficients in hybrid and mixed methods for nonlinear parabolic problems, Mat. Apl. Comput. 10 (1991), 137-160. MR 93d:65097

14. _ Prismatic mixed finite elements for second order elliptic problems, Calcolo 26 (1989), 135-148. MR 92e:65148

15. P. Ciarlet, The Finite Element Method for Elliptic Problems, North-Holland, Amsterdam, 1978. MR 58:25001

16. L. Cowsar, Dual-variable Schwarz methods for mixed finite elements, Dept. Comp. and Appl. Math. TR 93-09, Rice University, 1993. 
17. __ Domain decomposition methods for nonconforming finite element spaces of Lagrange type, in the Proceedings of the Sixth Copper Mountain Conference on Multigrid Methods, N. Melson et al., eds., NASA Conference Publication 3224 Part 1 (1993), 93-109.

18. L. Cowsar, J. Mandel, and M. Wheeler, Balancing domain decomposition for mixed finite elements, Dept. Comp. and Appl. Math. TR 93-08, Rice University, 1993.

19. L. Cowsar and M. Wheeler, Parallel domain decomposition method for mixed finite elements for elliptic partial differential equations, Proceedings of the Fourth International Symposium on Domain Decomposition Methods for Partial Differential Equations, R. Glowinski et al. eds., SIAM, 1991. CMP 91:12

20. Y. De Roeck and P. Le Tallec, Analysis and test of a local domain decomposition preconditioner, Proceedings of the Fourth International Symposium on Domain Decomposition Methods for Partial Differential Equations, R. Glowinski et al., eds., SIAM, 1991. CMP 91:12

21. J. Douglas, Jr., P. J. Paes Leme, J. E. Roberts, and J. Wang, A parallel iterative procedure applicable to the approximate solution of second order partial differential equations by mixed finite element methods, Numer. Math. 65 (1993), 95-108. MR 94c:65134

22. J. Douglas, Jr. and J. Wang, A new family of mixed finite element spaces over rectangles, Mat. Apl. Comput. 12 (1993), 183-197. CMP 94:16

23. M. Dryja and O. Widlund, Domain decomposition algorithms with small overlap, SIAM J. Sci. Statist. Comput. 15 (1994), 604-620. MR 95d:65102

24. R. Ewing, R. Lazarov, T. Russell, and P. Vassilevski, Local refinement via domain decomposition techniques for mixed finite element methods withrectangular Raviart-Thomas elements, Proc. Third Int. Symp. on DD Methods for PDE's, T. Chan et al., eds., SIAM, Philadelphia, 1990, pp. 98-114. MR 91f:65191

25. R. Ewing and J. Wang, Analysis of the Schwarz algorithm for mixed finite element methods, RAIRO Modél. Math. Anal. Numér. 26 (1992), 739-756. MR 94c:65135

26. Analysis of multilevel decomposition iterative methods for mixed finite element methods, RAIRO Modél. Math. Anal. Numér 28 (1994), 377-398. MR 95e:65099

27. R. Glowinski, W. Kinton, and M. Wheeler, Acceleration of domain decomposition algorithms for mixed finite elements by multilevel methods, Proceedings of the Third International Symposium on Domain Decomposition Methods for Partial Differential Equations, R. Glowinski et al., eds., SIAM, 1990, pp. 263-290. CMP 90:15

28. R. Glowinski and M. Wheeler, Domain decomposition and mixed finite element methods for el liptic problems, Domain Decomposition Methods for Partial Differential Equations, R. Glowinski et al., eds., SIAM, 1988, pp. 144-172. MR 90a:65237

29. J. Mandel, Balancing domain decomposition, Comm. Numer. Methods Engrg. 9 (1993), 233241. MR 94b:65158

30. J. Mandel and M. Brezina, Balancing domain decomposition: theory and performance in two and three dimensions, to appear.

31. T. P. Mathew, Schwarz alternating and iterative refinement methods for mixed formulations of elliptic problems, part I: algorithms and numerical results, Numer. Math. 65 (1993), 445468. MR 94m:65171

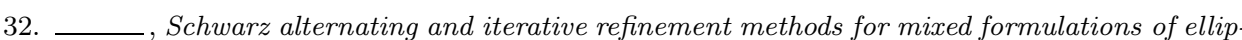
tic problems, part II: convergence theory, Numer. Math. 65 (1993), 469-492. MR 94m:65172

33. F. Milner, Mixed finite element methods for quasilinear second-order elliptic problems, Math. Comp. 44 (1985), 303-320. MR 86g:65215

34. J. C. Nedelec, Mixed finite elements in $\mathbf{R}^{3}$, Numer. Math. 35 (1980), 315-341. MR 81k:65125

35. A new family of mixed finite elements in $\mathbf{R}^{3}$, Numer. Math. 50 (1986), 57-81. MR 88e:65145

36. P. A. Raviart and J. M. Thomas, A mixed finite element method for 2 nd order elliptic problems, Mathematical Aspects of the Finite Element Method, Lecture Notes in Mathematics 606, Springer-Verlag, Berlin, 1977, pp. 292-315. MR 58:3547

37. M. Sarkis, Two-level Schwarz methods for nonconforming finite elements and discontinuous coefficients, in Proceedings of the Sixth Copper Mountain Conference on Multigrid Methods, N. Melson et al., eds., NASA Conference Publication 3224 Part 2 (1993), 543-566.

38. B. Smith, An optimal domain decomposition preconditioner for the finite element solution of linear elasticity problems, SIAM J. Sci. Statist. Comput. 13 (1992), 364-378. 
39. P. Vassilevski and J. Wang, An application of the abstract multilevel theory to nonconforming finite element methods, SIAM J. Numer. Anal. 32 (1995), 235-248. CMP 95:07

40. __ Multilevel iterative methods for mixed finite element discretizations of elliptic problems, Numer. Math. 63 (1992), 503-520. MR 93j:65187

Department of Mathematics and the Institute for Scientific Computation, Texas A\&M University, College Station, TX 77843

Current address, Z. Chen: Department of Mathematics, Box 156, Southern Methodist University, Dallas, Texas 75275-0156

E-mail address: zchen@isc.tamu.edu

E-mail address: ewing@ewing.tamu.edu

E-mail address: lazarov@math.tamu.edu 